\title{
Non-gradient, Sequential Algorithm for Simulation of Nascent Polypeptide Folding
}

\author{
Lech Znamirowski \\ Institute of Informatics, Silesian University of Technology, \\ ul. Akademicka 16, 44-100 Gliwice, Poland \\ lznamiro@top.iinf.polsl.gliwice.pl
}

\begin{abstract}
In the paper, the method for determining of the conformation of nascent protein folding based on two-phase, sequential simulation approach is presented. In both phases the potential energy of molecule under construction is minimized, however in the first phase the minimization is performed for the structure $\{$ new amino acid $\} /\{$ existing amino acid chain $\}$ and in the second phase, the $\{$ new existing amino acid chain $\}$ conformation is "tuned" to reach the minimal potential energy of growing chain. The formed, nascent conformation of protein determines initial condition for a future conformation modifications and plays a crucial role in fixing the biological and chemical features of created protein. The simulation of conformation process is illustrated through the numerical example of nascent protein folding for a selected protein.
\end{abstract}

\section{Introduction}

The chain of amino acids synthesized in a ribosome forms a polypeptide and in case of a long chain, a protein. For the conformation simulation tasks, the synthesis programs generating the conformation of the main chain of amino-acid residues in a polypeptide, use the amino acids library and need the information on the angles between $\alpha$-carbons bonded with their side chains and contiguous peptide groups [1, 2, 3]. The backbone conformation of the chain of amino-acid residues can be determined by the set of torsion angles [5] $\varphi$ (rotation around the nitrogen- $\alpha$-carbon bond), and $\psi$ (rotation around the $\alpha$-carbon-carbon bond in the chain of the polypeptide).

\subsection{Torsion Angles}

The relationship between the peptide groups, $\alpha$-carbons and torsion angles can be expressed in the following form

$$
\rightarrow \mathrm{PB} \rightarrow \phi_{1} \rightarrow \mathrm{C}_{\alpha 1} \rightarrow \psi_{1} \rightarrow \mathrm{PB} \rightarrow \phi_{2} \rightarrow \mathrm{C}_{\alpha 2} \rightarrow \psi_{2} \rightarrow \mathrm{PB} \rightarrow
$$

where PB denotes the peptide bond and $\mathrm{C}_{\alpha i}$ is the $i$-th $\alpha$-carbon atom. The torsion angles play a crucial role in the conformation of proteins (Fig. 1) because the threedimensional structure of protein determines its biological functions including its activity in a signal transduction cascades $[6,7,8]$. 
On the other hand not all combinations of torsion angles $\varphi_{i}$ and $\psi_{i}$ in (1) for each amino acid are possible, as many leads to collision between atoms in adjacent residues exposed by Ramachandran restrictions (map) [7, 9]. By assuming that atoms behave as hard spheres, allowed ranges of torsion angles can be predicted and visualized in contour of Ramachandran plots based on X-ray crystallography [10]. Nearly every polypeptide or protein in a cell is chemically altered after its synthesis in a ribosome. Many proteins are later modified in a reversible manner, fulfilling its functions after the activation forced by the messenger molecules. Such modifications expressed in the conformational changes $[11,12,13]$, may alter the biological activity, life span, or cellular location of proteins, depending on the nature of alteration.

\subsection{Conformational Energy}

The force powered changes of spatial shape is a tendency of the chain of polypeptide to get minimal potential energy. The potential energy of nanostructure defined also as conformational energy, according to the principles of molecular mechanics $[17,21$, 23] accepts the general form

$$
E(\mathbf{x})=\sum_{j} U_{j}(\mathbf{x}),
$$

where the expression $U_{j}(\mathbf{x})$ describes the proper type of potential energy (bonds stretching energy around equilibrium length of bonds, angles bending energy, multipole interactions energy, Lennard-Jones or generally Mie potentials etc.) of the intermolecular interactions there be a function of degrees of freedom and the physicochemical constants determined for the specified types of atoms, bonds, and in molecule configurations. The entries $x_{1}, x_{2}, x_{3}, \ldots$. of a vector $\mathbf{x}$ represent the degrees of freedom relating with distances and proper characteristic angles.

The spatial structure of molecule is described in one of the accepted standard formats: PDB [28] or mmCIF [27]. Dependent on type of atoms, type of bonds and the configuration of atoms in a molecule, the physicochemical constants are collected in tables (so called Force Field) e.g. AMBER [26], ENCAD [22], CHARMM [25] and others (depending on type of chemical compounds), applied in computations [16].

Minimizing the expression (2) which describes a conformational energy of structure for vector $\mathbf{x}$ with constraints (Ramachandran plots), it is possible to find a stationary point defining the stable structure in a given conditions. However, the complex form of expression (2), usually leads to the problem related with multiple local minima or saddle points. In the saddle points of the surface of energy for which the gradient $E(\mathbf{x})$ reaches zero the crucial role play the eigenvalues of hessian matrix built for function $E(\mathbf{x})$, which allow to define the farther run of the settlement of nanostructure conformation. And so simple algorithms of optimization e.g. gradient-based, Newton's or Fletcher-Powell have to be modified to find the global minimum. The implementation of algorithms of dynamic programming [3, 19, 20] or Monte Carlo methods $[4,15,24]$ is better solution.

Using new, two-phase sequential simulation approach, we will find the free conformation (initial conformation or nascent protein folding) for the exemplary protein 
through the minimization of the energy [16] as a function of the torsion angles $[3,16$, 17], however, we do not look for the global minimum of conformational energy, rather we perform the sequence of optimization corrections [14] for the growing chain. The simulation process retains the same sequence of events as a real process of nascent protein elongation.

\section{Two-Phase Sequential Simulation Algorithm}

The basic assumption for the construction of simulation algorithm is an observation, that the new amino-acid appearing from the ribosome rotates the existing chain of amino acids in a such manner, that the torsion angles on the new peptide bond, accordingly with minimization of potential energy of the structure \{new amino acid\} I \{existing amino acid chain $\}$ are determined, and next, the conformation of the whole structure is somewhat modified to minimize the potential energy of \{new existing amino acid chain $\}$. The first minimization plays a crucial role because of dimensions of peptide chain in comparison with the new molecule attached.

\subsection{Initialization}

The space defining available regions of $\varphi$ and $\psi$ angles (Fig. 1) is quantized and translated to the linear vector of available coordinates. The two first amino acids adopt the conformation minimizing expression

$$
\min _{\varphi_{0}, \psi_{0}, \varphi_{1}, \psi_{1}} E_{2}\left(\varphi_{0}, \psi_{0}, \varphi_{1}, \psi_{1}\right),
$$

where $E_{2}$ is a potential energy of the system of two amino acids, $\varphi_{0}$ and $\psi_{0}$ are the torsion angles of the first amino acid, as well as $\varphi_{1}$ and $\psi_{1}$ are the torsion angles of the second amino acid (Fig. 1a). In the Initialization and First Phase of simulation the search for energy decreasing is performed in the discretized, allowed ranges of torsion angles for glycine, proline and all 18 remaining amino acids specifically, predicted as modified Ramachandran plots based on X-ray crystallography [10]. In the Second Phase of simulation during the chain "shaking" process, only the generated torsion angles which remain in the allowed ranges are used. The energy computations apply the AMBER force field and the Tinker subroutines [16].

\subsection{First Phase of Simulation}

When the third residue appears (\#2 in Fig. 1b), the following expression is minimized

$$
\min _{\varphi_{2}, \psi_{2}} E_{3}\left(\varphi_{2}, \psi_{2}, \varphi_{0 f}, \psi_{0 f}, \varphi_{1 f}, \psi_{1 f}\right),
$$

where $E_{3}$ is a potential energy of the system of three amino acids, $\varphi_{0 f}, \psi_{0 f}, \varphi_{1 f}$, $\psi_{1 f}$ are the torsion angles determined in the initialization.

The first phase for fourth amino acid is preceded by the second phase which tunes the existing, whole chain to get the minimal potential energy (point 2.3).

Accordingly, for the fourth amino acid we minimize the expression 


$$
\min _{\varphi_{3}, \psi_{3}} E_{4}\left(\varphi_{3}, \psi_{3}, \varphi_{2 s}, \psi_{2 s}, \varphi_{0 f s}, \psi_{0 f s}, \varphi_{1 f s}, \psi_{1 f s}\right),
$$

where $E_{4}$ is a potential energy of the system of four amino acids, and $\varphi_{2 s}, \psi_{2 s}, \varphi_{0 f s}$, $\psi_{0 f s}, \varphi_{1 f s}, \psi_{1 f s}$ are the torsion angles determined in the second phase for the three amino acids (Fig. 1c).

\subsection{Second Phase of Simulation}

For the consecutive residues, bonded to the growing chain, the global tuning after the first phase takes place. Along the backbone of the chain (Fig. 1b, c), the torsion angles are changed in the four directions: $+k \Delta \varphi_{i},-k \Delta \varphi_{i},+k \Delta \psi_{i}$, and $-k \Delta \psi_{i}$, including the current $\varphi_{i}$ and $\psi_{i}(i=0,1, \ldots n)$ angles. Values of $\Delta \varphi_{i}$ and $\Delta \psi_{i}$ determine the amplitudes of reversible changes of torsion angles along the chain, and $k$ is a positive and decreasing parameter during the consecutive steps of the minimization of potential energy of the chain.

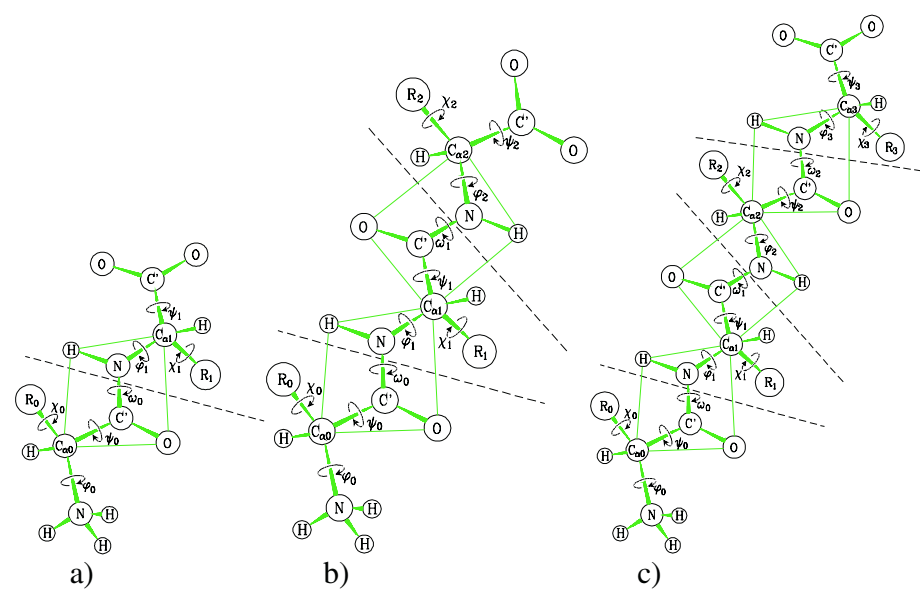

Fig. 1. Process of polypeptide elongation: a) initialization of simulation, b) the three residues (first phase of simulation), c) the four residues (first phase of simulation)

The parameter $k$ can be constant in one iteration step, as well as may be a function of selected parameters. In the proposed approach, we omit the problem of hydrogen and disulfide bonds creation.

\section{Numerical Example}

An illustration of presented two-stage simulation of nascent protein folding are the intermediate and final results in simulation of elongation process and conformational changes of the small protein containing thirty eight amino acids: 


$$
\begin{aligned}
& \text { asn - ser - tyr - pro - gly - cys - pro - ser - ser - tyr - asp - gly - tyr - cys - leu - } \\
& \text { asn - gly - gly - val - cys - met - his - ile - glu - ser - leu - asp - ser - tyr - thr - } \\
& \text { cys - asn - cys - val - ile - gly - tyr - ser. }
\end{aligned}
$$

The initialization phase of simulation based on the minimizati on of expression (3), gives the dipeptide asn-ser with four torsion angles. The conformation of dipeptide and the set of torsion angles is presented in Fig. 2.

When the third amino acid appears i.e. tyrosine, the first phase of simulation for the elongated tripeptide gives the results presented in Fig. 3a, b. The expression (4) is minimized over the $\varphi_{2}$ and $\psi_{2}$ angles with remaining angles unchanged.

In the second phase, the tripeptide chain is "shaked" along the backbone with $\Delta \varphi_{i}=\Delta \psi_{i}=32^{\circ}$ and $k$ decreasing twice per step until resolution $1^{\circ}$, better resolution usually needs parallel processing.

a)

$\begin{array}{crc}\text { Chain of } & \varphi_{i} & \psi_{i} \\ \text { amino acids } & & \\ \text { asn } & -60^{\circ} & -58^{\circ} \\ \text { ser } & -129^{\circ} & 141^{\circ}\end{array}$

b)

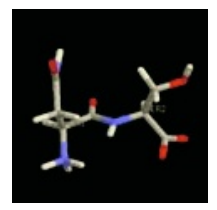

Fig. 2. The spatial shape of folded dipeptide asn-ser: a) the chain of torsion angles, b) RasMol [18] visualization of a molecule

The iterations stop when there is no decreasing value of the potential energy of tripeptide. As a result, the conformation of nascent tripeptide asn-ser-tyr gets the form presented in Fig. 3c, d.

a)

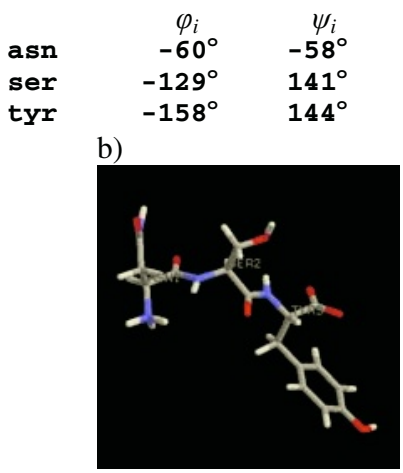

c)

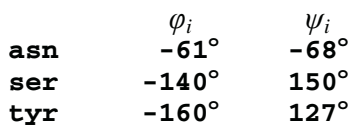

d)

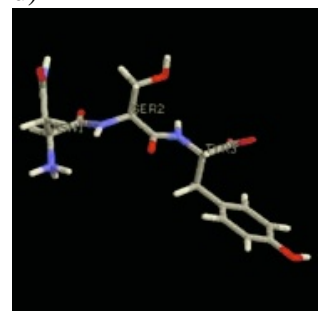

Fig. 3. The initial shape of folded tripeptide asn-ser-tyr: a) the chain of torsion angles, b) visualization of a molecule, and the final conformation of nascent tripeptide: c) torsion angles, d) visualization of a molecule 
Continuing this process, the eight amino acids chain obtains the conformation after the first and second phase of simulation presented in Fig. 4. Finally, the whole 38 amino acids chain adopts the conformation after first and second phase of simulation presented in Fig. 5. The results of computations have been compared with the results obtained in different discretization grid of the permissible torsion angles, and the differences were acceptable.

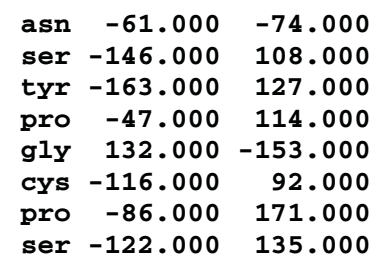

a)

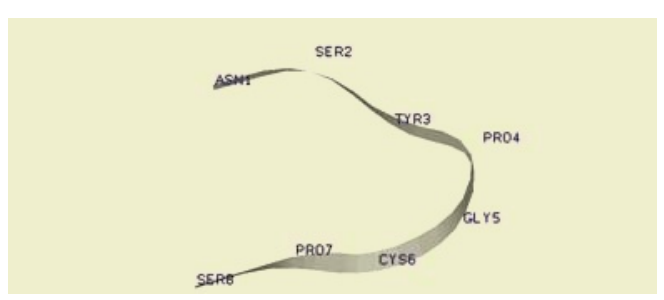

b)

Fig. 4. Final conformation of eight amino acids nascent protein after the second phase of simulation: a) torsion angles, b) backbone of molecule (ribbons visual representation [18])

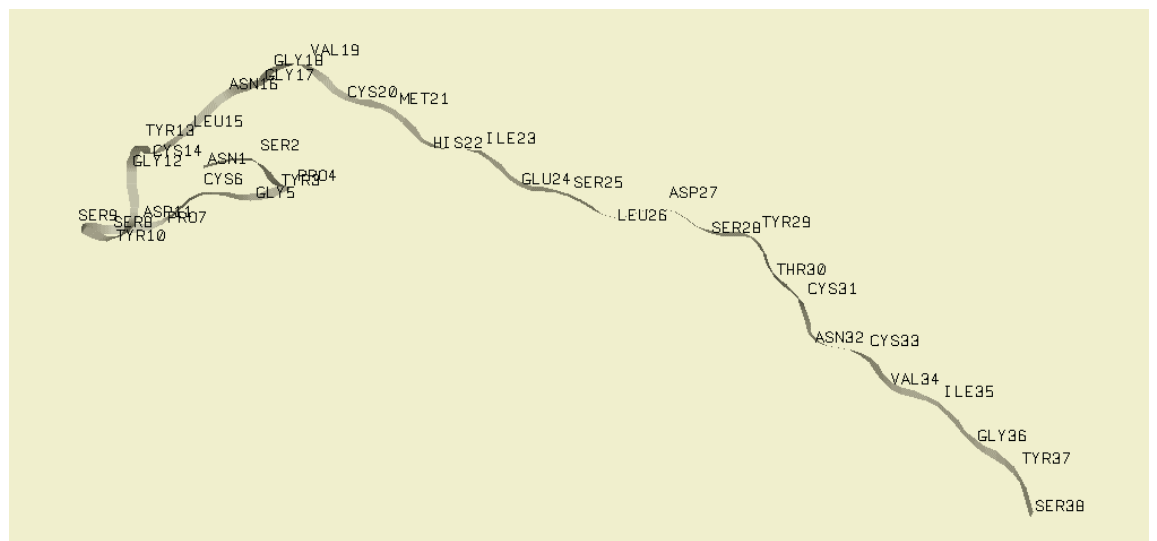

Fig. 5. Final conformation of 38 amino acids nascent protein after the second phase of simulation

The interesting observation can be do refer to the conformations of the same polypeptide sub-chain during the elongation phase and post-translational modifications namely cleavage of the main chain. In Fig. 6 the conformation of the initial eight amino acids cleaved from the whole chain has been presented. Comparing this conformation with the conformations of the same sub-chain of polypeptide in elongation phase (Fig. 4) we see the difference in the spatial shapes of these nanostructures. It suggests that the folding process of polypeptide depends on the sequence of external events preceding the final conformation. 


\section{Conclusions}

The paper presents two-phase algorithm for modeling and simulation of composite phenomena in protein processing: model building and simulation of initial phase of establishing the conformation of nascent protein folding.

The introductory experiments reveal, that the crucial role plays the phase when the new, consecutive amino acid bonds to the existing chain of polypeptide. This phenomenon is represented in simulation by the first phase, underlying fact that the new amino acid is partly fixed in the ribosome.

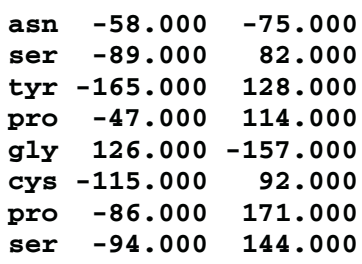

a)

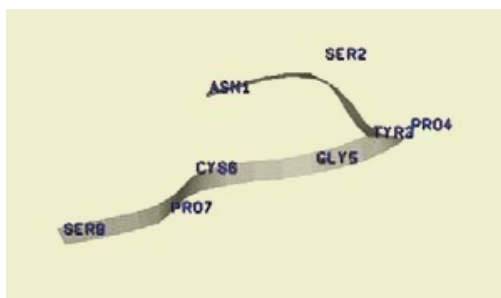

b)

Fig. 6. Conformation of the initial eight amino acids cleaved from the whole 38 amino acids nascent protein: a) torsion angles, b) backbone of molecule (compare with Fig. 4)

We can expect, that the similar situation appears at the output of chaperones [29]. In the second phase of simulation, the whole structure is under the influence of annealing process reaching the nascent conformation. This phase of protein folding can be treated as a correction of initial conformation.

The very interesting observation was made refer to post-translational modifications. The conformations of the same sub-chain of polypeptide in elongation phase and after cleavage of the main chain are different. It suggests that the folding process of polypeptide can not be understand as a simple minimization process of conformational energy but depends on the sequence of events preceding the final state.

Developed simulation software implementing mainly paradigms of molecular mechanics will be enriched in the scope of detection of stabilizing bonds as well as in the analysis tools for tracking the environmental modifications of protein conformation.

\section{References}

1. Berman, H. M., et al.: The Protein Data Bank. Nucleic Acid Res. 1 (2001) 235-242

2. "Klotho: Biochemical Compounds Declarative Database - Alphabetical Compound List". (2001), http://www.ibc.wustl.edu/moirai/klotho/compound_list.html

3. Znamirowski, L., Zukowska, E. D.: Simulation of Environment-Forced Conformations in the Polypeptide Chains. Proc. of the ICSEE'03, 2003 Western MultiConf., The Soc. for Mod. and Sim. Int., Orlando, Florida, January 19-23 (2003) 87-91

4. Zieliński, R.: Monte Carlo Methods. WNT Publisher, Warsaw (1970) (in Polish) 
5. Liebecq, C. (ed.): The White Book. Biochemical Nomenclature and Related Documents. IUPAC-IUBMB Joint Commission 2nd edn. Portland Press, London (1992)

6. "Cell Signaling Technology Database: Pathways". Cell Sign. Techn. Inc., Beverly, MA. (2003) info@cellsignal.com

7. Stryer L.: Biochemistry. W. H. Freeman and Company, New York (1994)

8. Heater, S. H., Zukowska, E. D., Znamirowski, L.: Information Processing in the Signal Cascades of Nanonetworks. ICCE-10, Tenth Ann. Intern. Conf. on Composites/Nano Engr., Intern. Comm. for Composite Engr., New Orleans, Louisiana, July 20-26 (2003) 233-234

9. Ramachandran, G. N., Sasisekharan, V.: Conformation of polypeptides and proteins. Advanced Protein Chem., 23 (1968) 506-517

10. Hovmoller, S, Zhou, T., Ohlson, T.: Conformations of amino acids in protein. Acta Cryst., 5 (2002) 768-776

11. Baker, D.: A surprising simplicity to protein folding. Nature, 406 (2000) 39-42

12. Okamoto, Y.: Protein folding simulations and structure predictions. Computer Physics Comm., 142 (2001) 55-63

13. Wei, C., Zhang, Y., Yang, K.: Chaperone-Mediated Refolding of Recombinant Prochymosin. Journal of Protein Chemistry, 6 (2000) 449-456

14. Znamirowski, A. W., Znamirowski, L.: Two-Phase Simulation of Nascent Protein Folding. Proc. of the Fourth IASTED Intern. Conf. on MODELLING, SIMULATION, AND OPTIMIZATION MSO 2004, Kauai, Hawaii, August 17-19, 2004, ACTA Press, Anaheim-Calgary-Zurich (2004) 293-298

15. Warecki, S., Znamirowski, L.: Random Simulation of the Nanostructures Conformations. Intern. Conference on Computing, Communication and Control Technology, Proceedings Volume I, The Intern. Institute of Informatics and Systemics, Austin, Texas, August 14-17 (2004) 388-393

16. Ponder, J.: TINKER - Software Tools for Molecular Design. Dept. of Biochemistry \& Molecular Biophysics Washington University, School of Medicine, St. Louis (2001) http://da sher.wustl.edu/tinker

17. Hinchliffe, A.: Modelling Molecular Structures. 2nd edn. John Wiley \& Sons, Ltd, Chichester (2000)

18. Sayle, R.: RasMol, Molecular Graphics Visualization Tool. Biomolecular Structures Group, Glaxo Wellcome Research \& Development, Stevenage, Hartfordshire (1998) H. J. Bernstein v.2.7.1.1, rasmol@ bernstein-plus-sons.com

19. Bellman, R.: Dynamic Programming. Princeton University Press, Princeton, N. J. (1957)

20. Węgrzyn, S., Winiarczyk, R., Znamirowski, L.: Self-replication Processes in Nanosystems of Informatics. Int. Journ. of Applied Math. and Comp. Science, Vol. 13, 4 (2003) 585-591

21. Atkins, P. W.: Physical Chemistry, 6th edn. W. H. Freeman \& Co., New York (1998)

22. Levitt, M., Hirshberg, M., Sharon, R., Daggett, V.: Potential Energy Function and Parameters for Simulation of the Molecular Dynamics of Protein and Nucleic Acids in Solution. Comp. Physics Comm., 91 (1995) 215-231

23. McMurry, J.: Organic Chemistry, 4th edn. Brooks/Cole Publ. Co., Pacific Grove (1996)

24. Metropolis, N., Ulam, S.: The Monte Carlo Method. Journal of the American Stat. Assoc., Vol. 44, 247 (1949) 335-341

25. MacKerrell, A. D. et al.: All-Atom Empirical Potential for Molecular Modeling and Dynamics Studies of Proteins. Journ. Phys. Chem. B, 102 (1998) 3586-3616

26. Cornell, W. D. et al.: A Second Generation Force Field for the Simulation of Proteins, Nucleic Acids, and Organic Molecules. Journ. Am. Chem. Soc., Vol. 117 (1995) 5179-5197 
27. "The Nucleic Acid DataBase Project". Rutgers, The State University of New Jersey, Camden, Newark, New Brunswick/Piscataway (2001), http://ndb-mirror-2.rutgers.edu/mmcif/ index.html

28. Callaway, J., Cummings, M. et al.: Protein Data Bank Contents: Atomic Coordinate Entry Format Description. Federal Govern. Agency (1996), http://www.rcsb.org/pdb/docs/ format/ pdbguide2.2/

29. Lodish, H., Berk, A., Zipursky, S. L., et al.: Molecular Cell Biology, 4th edn. W. H. Freeman and Comp., New York (2001) 